\title{
Is it possible to manipulate root anchorage in young trees?
}

\author{
Hayfa Khuder · Alexia Stokes · Frédéric Danjon • \\ Kyriaki Gouskou · Frédéric Lagane
}

Published online: 26 May 2007

(C) Springer Science+Business Media B.V. 2007

\section{Erratum to: Plant Soil (2007) DOI 10.1007/s11104-007-9232-6}

Table 2 "Specific root surface $\left(\mathrm{m}^{-2}\right)$ " should be "Specific root surface $\left(\mathrm{m}^{-1}\right)$,"

The online version of the original article can be found under doi: 10.1007/s11104-007-9232-6

\section{H. Khuder}

Université Bordeaux I, INRA, CNRS, UMR US2B,

Talence 33405, France

e-mail: khuder@1rbb3.pierroton.inra.fr

A. Stokes

INRA, LIAMA-CASIA, P.O. Box 2728, Haidian District, 100080 Beijing, China

Present Address:

A. Stokes $(\square)$

INRA, Botanique et Bioinformatique de l'Architecture des Plantes (AMAP), TA A-51/PS2, Boulevard de la Lironde, Montpellier Cedex 5 34398, France e-mail: stokes@1rbb3.pierroton.inra.fr

F. Danjon · K. Gouskou · F. Lagane INRA, UR1263 EPHYSE, 69 route d'Arcachon, Cestas 33612, France

F. Danjon

e-mail:danjon@pierroton.inra.fr

K. Gouskou

NAGREF, Forest Research Institute, Vassilika,

Thessaloniki 57006, Greece
"'Specific root length $\left(\mathrm{m}^{-1}\right)$, should be "Specific root length $\left(\mathrm{m}^{-2}\right)$," 
Table 2 Mean root growth parameters \pm standard error for the 76 cuttings and trees with (+) or without (-) tap roots

\begin{tabular}{|c|c|c|c|c|}
\hline \multirow[t]{2}{*}{ Growth parameter } & \multicolumn{3}{|l|}{ Treatment } & \multirow[t]{2}{*}{$P$} \\
\hline & Cutting & (-) Tap root & (+) Tap root & \\
\hline $\mathrm{RPC}(\%)$ & $0.20 \pm 0.08$ & $0.20 \pm 0.09$ & $0.18 \pm 0.11$ & 0.209 \\
\hline Total root volume $\left(\mathrm{m}^{3}\right)$ & $0.009 \pm 0.001^{\mathrm{a}}$ & $0.014 \pm 0.002^{\mathrm{b}}$ & $0.018 \pm 0.003^{\mathrm{b}}$ & 0.003 \\
\hline Total root length (m) & $9.7 \pm 1.0^{\mathrm{a}}$ & $19.9 \pm 1.0^{\mathrm{b}}$ & $22.7 \pm 2.0^{\mathrm{b}}$ & $<0.001$ \\
\hline Specific root surface $\left(\mathrm{m}^{-1}\right)$ & $360 \pm 20^{\mathrm{a}}$ & $430 \pm 30^{\mathrm{b}}$ & $400 \pm 30^{\mathrm{a}, \mathrm{b}}$ & $<0.001$ \\
\hline Specific root length $\left(\mathrm{m}^{-2}\right)$ & $16000 \pm 2000^{\mathrm{a}}$ & $24000 \pm 3000^{\mathrm{b}}$ & $20000 \pm 3000^{\mathrm{a}, \mathrm{b}}$ & $<0.001$ \\
\hline Maximal radial distance $(\mathrm{m})$ & $1.4 \pm 0.8^{\mathrm{a}}$ & $1.5 \pm 0.8^{\mathrm{a}, \mathrm{b}}$ & $1.6 \pm 1.0^{\mathrm{b}}$ & 0.124 \\
\hline Maximal depth (m) & $0.7 \pm 0.2$ & $0.7 \pm 0.3$ & $0.7 \pm 0.2$ & 0.216 \\
\hline Tap root length $(\mathrm{m})$ & $0.8 \pm 0.3$ & $0.8 \pm 0.5$ & $0.8 \pm 0.4$ & 0.792 \\
\hline Relative root number (number/DBH (m)) & $389.9 \pm 16.2^{\mathrm{a}}$ & $578.0 \pm 39.1^{\mathrm{b}}$ & $564.7 \pm 22.7^{\mathrm{b}}$ & $<0.001$ \\
\hline Mean number of lateral roots on stump & $8.8 \pm 0.6^{\mathrm{a}}$ & $20.5 \pm 1.2^{\mathrm{b}}$ & $19.6 \pm 1.4^{\mathrm{b}}$ & $<0.001$ \\
\hline Mean basal diameter of lateral roots on stump (mm) & $17.0 \pm 0.1^{\mathrm{a}}$ & $13.1 \pm 0.1^{\mathrm{b}}$ & $14.7 \pm 0.1^{\mathrm{a}, \mathrm{b}}$ & $\mathbf{0 . 0 3 1}$ \\
\hline Mean inter-lateral length along tap root (mm) & $51.6 \pm 3.9^{\mathrm{a}}$ & $29.3 \pm 3.1^{\mathrm{b}}$ & $27.1 \pm 2.1^{\mathrm{b}}$ & $<0.001$ \\
\hline Proportion of stump volume $(\%)$ & $49.3 \pm 1.6$ & $49.6 \pm 1.3$ & $46.5 \pm 1.1$ & 0.128 \\
\hline Root wood density $\left(\mathrm{kg} / \mathrm{m}^{3}\right)$ & $422.8 \pm 7.7^{\mathrm{a}}$ & $454.9 \pm 19.5^{\mathrm{b}}$ & $456.7 \pm 7.6^{\mathrm{b}}$ & 0.050 \\
\hline Shallow soil layer (all root segments) $(\%)$ & $69.0 \pm 2.9$ & $65.6 \pm 4.7$ & $61.8 \pm 2.8$ & 0.33 \\
\hline Intermediate soil layer (all root segments) (\%) & $29.9 \pm 2.7$ & $31.7 \pm 4.3$ & $35.9 \pm 2.6$ & 0.41 \\
\hline Deep soil layer (all root segments) (\%) & $1.1 \pm 0.3$ & $2.1 \pm 0.6$ & $1.9 \pm 0.5$ & 0.12 \\
\hline
\end{tabular}

Proportion of root volume (\%) between different compartments (with stump removed)

\begin{tabular}{lcccc} 
Horizontal shallow segments within ZRT & $17.1 \pm 2.0$ & $15.8 \pm 2.0$ & $15.8 \pm 1.6$ & 0.86 \\
Intermediate roots & $6.2 \pm 1.8$ & $5.1 \pm 2.2$ & $4.3 \pm 1.1$ & 0.76 \\
Oblique roots & $12.4 \pm 2.4^{\mathrm{a}}$ & $4.6 \pm 1.6^{\mathrm{b}}$ & $9.1 \pm 1.9^{\mathrm{a}, \mathrm{b}}$ & 0.09 \\
Deep roots & $0.37 \pm 0.14$ & $0.42 \pm 0.21$ & $0.56 \pm 0.17$ & 0.74 \\
Tap root & $27.8 \pm 2.2$ & $26.1 \pm 3.1$ & $22.8 \pm 2.4$ & 0.24 \\
Sinker roots beneath ZRT & $11.2 \pm 1.9^{\mathrm{a}}$ & $18.1 \pm 2.4^{\mathrm{b}}$ & $17.5 \pm 2.6^{\mathrm{b}}$ & 0.06 \\
Sinker roots beyond ZRT & $0.5 \pm 0.4$ & $0.6 \pm 0.3$ & $0.4 \pm 0.2$ & 0.56 \\
Horizontal shallow segments beyond ZRT & $25.2 \pm 2.4$ & $30.0 \pm 3.2$ & $31.1 \pm 2.1$ & 0.22 \\
\hline
\end{tabular}

Where letters in superscript differ, differences are significant between treatments using a Fisher LSD test $(P<0.05)$. In the Probability $(P)$ column, $P$ values (significant $P$ values are in bold) are given for the comparison of all three treatments together using ANOVA (with DBH as covariate). Non-proportional data were log-transformed and proportional data were arcsine square root transformed before analysis, although mean values of raw data are given here for ease of reading 
Legend of Fig. 2 should read:

(a)

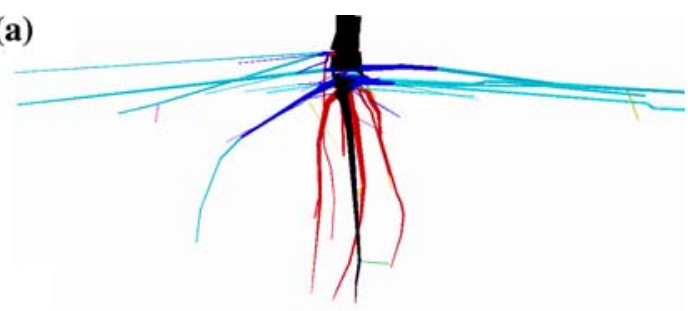

(b)

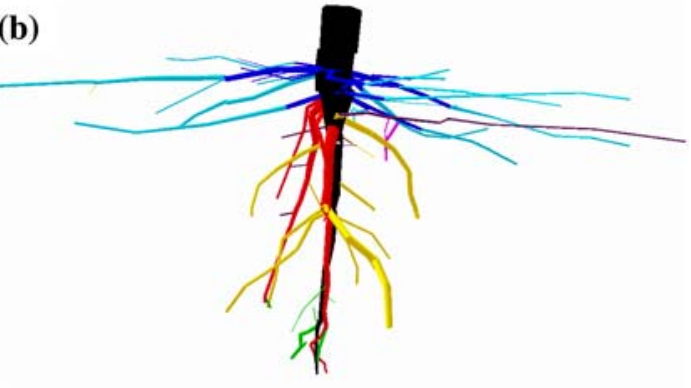

(c)

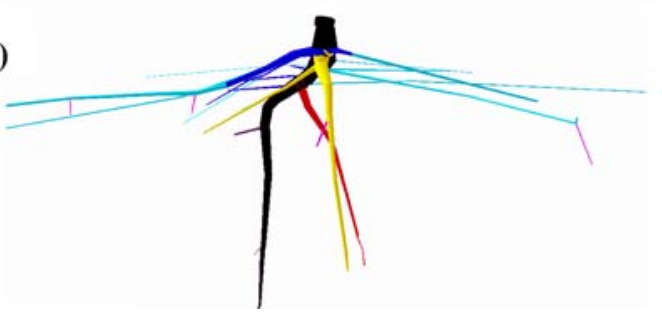

Fig. 2 Reconstruction with AMAPmod of root systems coloured as a function of compartment type in (a) trees with pruned tap roots, (b) control trees with undamaged tap roots, and (c) cuttings. The root systems are at the same scale with a maximal rooting depth of $-0.75,-0.9$, and $-0.8 \mathrm{~m}$, respectively. Side view, left is North West 